\title{
Efficacy and Safety of Fluvastatin Sodium XL 80mg in Treatment of Hypercholesterolemic Patient with Risk Factor of Cardiovascular Disease
}

\author{
FTN Malik1, M Badiuzzaman¹, MN Ahmed ${ }^{1}$, MS Haque ${ }^{2}$, MS Azam³ ${ }^{3}$ MJ Uddin³, \\ MA Khaleq ${ }^{4}$, BU Ahmed ${ }^{5}$, P Bhattacharjee ${ }^{6}$, MNA Khan ${ }^{7}$, GA Mamun ${ }^{8}$ \\ ${ }^{1}$ National Heart Foundation Hospital \& Research Institute, ${ }^{2}$ Dhaka Medical College and Hospital, \\ ${ }^{3}$ Chittagong Medical College Hospital, ${ }^{4}$ Cardiology, Modernized Hospital , Joypurhat, ${ }^{5}$ Debidwar \\ Thana Health Complex, Comilla, ${ }^{6}$ Asian Cardiac \& General Hospital, Dhaka, ${ }^{7}$ Chittagong Diabetic \\ Hospital , Chittagong, ${ }^{8}$ Dr. MAMUN's Diabetes Specialities Centre, Chittagong.
}

Key words: Hypercholesterolemia, Dyslipidemia, Lipid lowering agent, Fluvastatin.

\begin{abstract}
:
Back ground: Reduction of coronary heart disease (CHD) risk through the modification of risk factors has a strong effect on clinical practice. The introduction of 3-hydroxy-3-methylglutaryl coenzyme-A (HMG-CoA) reductase inhibitors (statins) has significantly advanced the treatment of hypercholesterolemia and in reduction of cardiovascular events and total mortality rates. Among the available statins, Fluvastatin is a newer, synthetic, second generation, potent lipid lowering agent and widely accepted in diverse population. However the safety profile and efficacy was not assessed in Bangladeshi population, a population significantly different from Caucasian population where most studies were done. Current study aimed at evaluating the safety and efficacy of fluvastatin in the specified population.
\end{abstract}

Methods: The study is an open-label, multicenter, quasi experimental study conducted among 162 adult patients suffering from hypercholesterolemia. After through baseline evaluation, the patients were given with Fluvastatin $80 \mathrm{mg}$ once daily for 3 months. All the patients were assessed twice, before and after treatment. Data on demography, of relevant medical history and of physical examination were collected in the both the visit along with data on relevant lipid parameters (Total Cholesterol, LDL-C, HDL-C and TG) were collected at final visit. Safety was assessed by evaluating adverse events, as well as laboratory abnormalities, including liver aminotransferases.

Results: Serum total cholesterol was found to be significantly reduced and across two assessments the reduction was 51.2 units $(P<.001)$. Average reduction in $L D L$-cholesterol was around 40 units

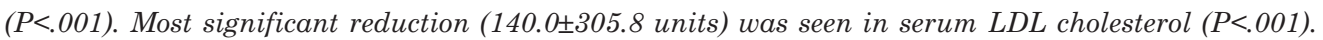
However; no statistically significant reduction was seen in HLD cholesterol. Safety of fluvastatin was assessed by evaluating the adverse events, as well as through laboratory abnormalities, including alanine aminotransferase $(A L T)$ and aspartate aminotransferase (AST). Comparison of aminotransferase level was done before and after treatment through paired t test, Neither ALT nor the AST showed statistically significant rise after 3 months treatment of fluvastatin $(P>.05)$. Out of 162 study participant $4.3 \%$ had their treatment interrupted, of which $1(0.62 \%)$ had to cease treatment due to lack of efficacy, $1(0.62 \%)$ experienced adverse event, $2(1.24 \%)$ didn't return to follow-up and $3(1.86 \%)$ patients requested their physician to cease the treatment.

Conclusion: Three month treatment with Fluvastatin XL $80 \mathrm{mg}$ reduces most of lipid parameter of lipid profile (Total cholesterol, Triglyceride and LDL) significantly. The drug is found to be well tolerated with minimal adverse event during the course of treatment.

(Cardiovasc. j. 2010; 2(2) : 147-155)

\section{Introduction:}

Atherosclerosis is considered as one the leading cause of death worldwide. ${ }^{1}$ The best strategy to decrease the death toll from NCDs such as Atherosclerosis and its consequences is yet to be established in many of the countries concerned. Decisive prevention policies are yet to be formulated and implemented, particularly in resource-limited developing countries. The increased risk of cardiovascular disease is

Address of Correspondence: Prof. Fazila-Tun-Nesa Malik, National Heart Foundation Hospital \& Research Institute. Dhaka. Bangladesh 
associated with an elevated serum cholesterol level ${ }^{2}$ and can be reduced by lowering the blood cholesterol concentration ${ }^{3}$.

There is now considerable evidence that controlling hypercholesterolemia can reduce the incidence of coronary heart disease. ${ }^{4}$ Both non pharmacologic and pharmacologic treatment are in practice. Standard non-pharmacological therapy consists primarily of modification of diet and lifestyle. This therapy may modestly reduce LDL cholesterol, but is not likely to lower the LDL cholesterol level more than about $30 \mathrm{mg} / \mathrm{dL} .{ }^{5}$ General physicians and heart specialists prescribes the medication in combination with diet and exercise. Pharmacological treatment should be started at an early age in order to prevent premature coronary artery disease. However, the treatment of hypercholesterolemia is occasionally difficult, as moderate hypercholesterolemia gives no symptoms, and the patients often experience sideeffects of several chole-sterol-lowering drugs. ${ }^{6}$ The ideal drug would be efficacious in lowering plasma lipid levels with no subjective side-effects, would have a documented effect on the clinical endpoint, and would be safe on a long-term basis. ${ }^{7}$

The introduction of HMG-CoA reductase inhibitors (statins) has significantly advanced the treatment of hypercholesterolemia. Statins can reduce LDL cholesterol levels by $20-40 \%$ and, at maximum doses; they can lower levels by $40-50 \%$. They also can modestly increase HDL cholesterol levels, usually by about $5-10 \%$. These medications are usually well tolerated, have few side effects. ${ }^{5}$

Published evidence in of recent observations shows the statins as the most commonly used and effective forms of medication for the treatment of elevated cholesterol. The U.S. Preventive Services Task Force estimated that after 5 to 7 years of treatment with statins, the relative risk reduction on coronary heart disease events was achieved by approximately $30 \% .{ }^{8,9}$ More recently, a systematic review reported an almost identical relative risk reduction of $29.2 \%$ in low risk patients treated for 4.3 years. ${ }^{10}$ Meta analysis by Bigen et al reported relative risk reduction of $19 \%$ in mortality due to coronary heart disease. ${ }^{11}$

From six available statins approved for patients requiring lipid-lowering therapy, Fluvastatin is a preferred one. Among the available statins
Fluvastatin is the only synthetic molecule, a second generation, potent competitive inhibitor of 3 hydroxy-3-methylglutaryl coenzyme-A reductase, the rate-limiting enzyme in cholesterol biosynthesis. When used in conjunction with a fatand cholesterol-restricted diet, it shows marked efficacy in treatment of hypercholesterolemia and mixed hypercholesterolemia. ${ }^{12}$ Several clinical outcome trials have shown that substantial benefits are associated with treatment with fluvastatin in diverse populations.

Research in Caucasian population suggests, with its safety profile and evidence of clinical benefits, fluvastatin can play an important role in the treatment of patients with hypercholesterolaemia. Fluvastatin XL has been shown to reduce LDLcholesterol significantly in high-risk patients to an extent similar to other statins, lovastatin ER, pravastatin and simvastatin at starting dosages, although to a lesser extent than atorvastatin and rosuvastatin. ${ }^{13}$

Because of its safety profile, fluvastatin represent an excellent choice for, patients needing moderate LDL-cholesterol reductions to achieve LDLcholesterol goals; for patients requiring combination therapy with other lipid-lowering agents; for patients taking multiple medications that place them at high risk for drug interactions during statin therapy; and high-risk patient populations, including patients with renal disease, renal transplantation patients and patients who have undergone coronary intervention procedures. ${ }^{13}$ Several clinical outcome trials have shown that substantial benefits are associated with treatment with fluvastatin in such diverse populations. In particular, data from two large, randomised clinical trials ${ }^{14,15}$ have demonstrated that fluvastatin is effective for secondary prevention of cardiac events in patients following coronary intervention procedures, and for primary prevention of cardiac events in renal transplant recipients.

However the safety profile and efficacy was not assessed in Bangladeshi population, a population significantly different from Caucasian, Negrito and other racial variations. The aim of the study was to evaluate the safety and efficacy of fluvastatin in the specified population and in its registered indication in treating patients with hyperlipidemia 
and or mixed dyslipidemia with risk factors of cardiovascular disease.

\section{Methodology:}

\section{Patients and materials:}

Current effort is an open-label, multicenter, quasi experimental study conducted among 162 adult patients suffering from hypercholesterolemia to evaluate the safety and efficacy of fluvastatin in Bangladesh population. The study was approved by the institutional review board and was conducted maintaining confidentiality. Initially a total of 169 patients were recruited upon their consent through their treating physician in 40 urban centres of Bangladesh. The assigned physicians were the registered doctors practicing in the urban area. Recruited subjects were adults with registered indication of fluvastatin, having hypercholesterolemia (total cholesterol $>200$ or $\mathrm{LDL} \geq 130 \mathrm{mg} / \mathrm{dl}$ ) and or mixed dyslipidemia with risk factors for Cardiovascular disease, namely, Hypertension ( $\mathrm{BP} \geq 140 / 90 \mathrm{~mm}$ of $\mathrm{Hg}$ or on antihypertensive medications), Low HDLCholesterol $(<40 \mathrm{mg} / \mathrm{dl})$, history of recent PCI and Diabetes mellitus. Patients with hypersensitivity to any of the components of fluvastatin, with chronic renal failure (with serum creatinine> $2.0 \mathrm{mg} / \mathrm{dL}$ ), Patient with active liver disease, or unexplained persistent elevations in serum transaminases and pregnant or nursing women were excluded from the study. After thorough baseline evaluation, the patient was given with Fluvastatin $80 \mathrm{mg}$ once daily. To maintain similarity in intervention all the patients were prescribed the investigational drug of same brand (LESCOL XL $^{\circledast}$ sustained release tablet containing $80 \mathrm{mg}$ fluvastatin). To avoid contamination of results, Patients having concomitant therapy with other antihyperlipidemics or drugs with the potential of confounding effect in judgment of the treating physician were dropped. Complete data was not available on 7 patients, as they didn't attend the final follow up assessment. Finally data of 162 subjects, who took the medication for 3 months as prescribed by the physician and attended follow up over the period and of whom complete data was available, were proceeded for final analysis
Assessment of baseline parameters, Outcome and study end points:

All the patients were assessed twice, once at the baseline and once upon completion of three month course of treatment. And the data were recorded in a predesigned Case Report Forms (CRF) by the treating physician. During the first visit, patients were screened for eligibility for enrollment based on the inclusion and exclusion criteria. Baseline data on demography, of relevant medical history and of Physical examination were collected in the visit along with data on relevant lipid parameters revealed through Laboratory investigations (Total Cholesterol, LDL-C, HDL-C and TG). At the end of 3 month therapy the second and final assessment was done repeating the Physical and Laboratory examinations done at baseline for comparison. In the visit additionally data on Safety of the drug was collected by inquiring and recording all adverse events or serious adverse events. An adverse event considered by the treating physician, in the study is any undesirable sign, symptom or medical condition occurring after starting study drug even if the event is not considered to be related to study drug. Information about all adverse events either self reported, or elucidated by the physician were recorded. A Serious adverse event considered for the study is an undesirable sign, symptom or medical condition which is fatal or life-threatening or required prolonged hospitalization or resulted in persistent or significant disability or incapacity or that it may jeopardize the subject and may require medical or surgical intervention.

\section{Statistical analysis:}

The data from all 40 centers were pooled and summarized with respect to demographic and baseline characteristics and efficacy and safety observations. Safety was assessed by summarizing frequencies of adverse events and Laboratory evaluation of liver aminotransfares. Adverse events were summarized by presenting the number and percentage of patients having any adverse event. Both post treatment lipid parameters and liver aminotransfares were compared with those of baseline through paired t test. McNemar test is done on $2 \times 2$ classification table for comparison of proportion of overweight and hypertension in before after setting. For statistical analysis Statistical software $\operatorname{SPSS}^{\circledast}$ version $16^{16}$ and STATA $^{\circledast}$ version $10 \mathrm{I} / \mathrm{C}^{17}$ were used. 
Results:

a. Patient characteristics:

Table-I

Distribution of the study subjects by age and sex

\begin{tabular}{lccc}
\hline Age group & Male & Female & Total \\
\hline$<40$ years & $14(12.5 \%)$ & $09(18.0 \%)$ & $23(14.2 \%)$ \\
40 - 49 years & $47(42.0 \%)$ & $20(40.0 \%)$ & $67(41.4 \%)$ \\
50 - 59 years & $38(33.9 \%)$ & $11(22.0 \%)$ & $49(30.2 \%)$ \\
$>=60$ years & $13(11.6 \%)$ & $10(20.0 \%)$ & $23(14.2 \%)$ \\
\hline Total & $112(100.0 \%)$ & $50(100.0 \%)$ & $162(100.0 \%)$ \\
\hline
\end{tabular}

Mean age Male $48.7 \pm 8.1$; Female $48.7 \pm 10.2$

Table 1 shows the distribution of the study participants by age and sex. Out of 162 participants
$112(69.1 \%)$ were male and $50(30.9 \%)$ were male. Mean age among Male was $48.7 \pm 8.1$ year and among Female was $48.7 \pm 10.2$ year. Among the study participants $14.2 \%$ were aged below 40 years, $41.4 \%$ were aged between $40-49$ years, $30.2 \%$ were aged between 50 - 59 years and $14.2 \%$ were aged above 60 years.

Number in parenthesis indicated column percentage, *Statistically significant at $\mathrm{P}<.05$, McNemar test is done on $2 \times 2$ classification table for comparison of proportion in before after setting.

Anthropometric measurements were recorded and BMI was calculated from height and weight recorded both at baseline and after treatment. Majority of the (82.1\%) participants were overweight at baseline and at the $2^{\text {nd }}$ visit the

Table-II

Comparison of BMI and Hypertension status before and after treatment

\begin{tabular}{|c|c|c|c|c|c|}
\hline \multirow[t]{2}{*}{$\overline{\text { Variables }}$} & \multicolumn{2}{|c|}{ Assessment time } & \multicolumn{2}{|c|}{ Paired difference } & \multirow[b]{2}{*}{$\mathrm{p}$ value } \\
\hline & Visit 1 & Visit 2 & proportions & $95 \% \mathrm{CI}$ & \\
\hline \multicolumn{6}{|l|}{ BMI Category } \\
\hline Normal weight(d" 24) & $29(17.9 \%)$ & $37(22.8 \%)$ & $4.94 \%$ & $-0.5 \%$ to $8.4 \%$ & 0.077 \\
\hline Over weight (>24) & $133(82.1 \%)$ & $125(77.2 \%)$ & & & \\
\hline \multicolumn{6}{|l|}{ Hypertension } \\
\hline Yes & $100(61.7 \%)$ & $152(93.8 \%)$ & $32.10 \%$ & $26.1 \%$ to $32.7 \%$ & $0.001^{*}$ \\
\hline No & 62 & $(38.3 \%)$ & $10(6.2 \%)$ & & \\
\hline
\end{tabular}

Table-III

Distribution of the study subjects by history of medical condition and concomitant medication ( $n=162)$

\begin{tabular}{lcc}
\hline Variables & Frequency & Percent \\
\hline Medical condition & 50 & \\
Yes & 112 & 30.9 \\
No & & 69.1 \\
Concomitant medication & 53 & 32.7 \\
Yes & 109 & 67.3 \\
No &
\end{tabular}

Table-IV

Comparison of lipid profile before and after fluvastatin treatment through paired t test

\begin{tabular}{|c|c|c|c|c|c|}
\hline \multirow[t]{2}{*}{ Variables } & \multicolumn{2}{|c|}{ Assessment time } & \multicolumn{2}{|c|}{ Paired mean difference } & \multirow[b]{2}{*}{$\mathrm{p}$ Value } \\
\hline & Visit 1 & Visit 2 & Difference & t Value & \\
\hline Total cholesterol $(\mathrm{n}=162)$ & $269.9 \pm 57.3$ & $218.1 \pm 40.6$ & $51.9 \pm 34.8$ & 18.94 & $.000 *$ \\
\hline Triglyceride(n=162) & $400.0 \pm 337.9$ & $260.4 \pm 115.5$ & $140.0 \pm 305.8$ & 05.83 & $.000 *$ \\
\hline $\operatorname{LDL}(\mathrm{n}=162)$ & $161.1 \pm 54.5$ & $121.1 \pm 33.2$ & $40.1 \pm 48.0$ & 10.62 & $.000 *$ \\
\hline $\operatorname{HDL}(\mathrm{n}=162)$ & $42.3 \pm 45.8$ & $42.11 \pm 25.5$ & $0.17 \pm 37.6$ & 00.06 & .953 \\
\hline
\end{tabular}

* Statistically significant; p value was generated through paired t test. 
Table-V

Comparison of aminotransferase before and after fluvastatin treatment through paired test

\begin{tabular}{lcccccc}
\hline Variables & \multicolumn{2}{c}{ Assessment time } & & \multicolumn{2}{c}{ Paired mean difference } & \\
\cline { 2 - 3 } & Visit 1 & Visit 2 & & difference & T value & p value \\
\hline ALT & $33.52 \pm 9.3$ & $33.10 \pm 8.0$ & & $.425 \pm 5.119$ & 0.53 & .602 \\
AST & $26.79 \pm 8.3$ & $25.21 \pm 7.2$ & & $1.583 \pm 4.127$ & 1.88 & .073 \\
\hline
\end{tabular}

Table-VI

Outcome assessment of treatment

\begin{tabular}{|c|c|c|c|}
\hline & Variables & Frequency & Percent \\
\hline \multirow[t]{4}{*}{1} & Interruption of treatment & & \\
\hline & Yes & 07 & 4.3 \\
\hline & No & 155 & 95.7 \\
\hline & Total & 162 & 100.0 \\
\hline \multirow[t]{5}{*}{2} & Reason for interruption & & \\
\hline & Lack of efficiency & 01 & 14.3 \\
\hline & Adverse event & 01 & 14.3 \\
\hline & Patient's request & 03 & 42.9 \\
\hline & Lost to follow up & 02 & 28.6 \\
\hline \multirow[t]{3}{*}{3} & Tolerance & & \\
\hline & Very good & 95 & 58.6 \\
\hline & Good & 67 & 41.4 \\
\hline \multirow[t]{4}{*}{4} & Success & & \\
\hline & Very good & 91 & 56.2 \\
\hline & good & 68 & 42.0 \\
\hline & Not satisfactory & 03 & 1.9 \\
\hline
\end{tabular}

proportion of overweight was reduced to $77.2 \%$, however the decrease was not statistically significant $(\mathrm{P}>.05)$. Blood pressure was measured at both the assessment, A systolic $\mathrm{BP}>140 \mathrm{mmHg}$ or diastolic $\mathrm{BP}>90 \mathrm{mmHg}$ was considered as hypertension. At the commencement of the study the proportion of hypertension measured was $38.3 \%$ and at the $2^{\text {nd }}$ visit the proportion dropped sharply to $6.2 \%$ and the decrease was highly significant $(\mathrm{P}<.01)$.

Table shows the distribution of the study subjects by presence of medical condition at base line and concomitant treatment during the drug therapy. Among the patients $30.9 \%$ had been suffering from any medical condition and $32.7 \%$ received concomitant medication while taking fluvastatin therapy.

\section{b. Efficacy of Fluvastatin}

Efficacy of fluvastatin treatment was assessed by assessing the lipid lowering capability of the drug by comparing after-treatment lipid profile with the baseline. The mean of difference in total cholesterol across two assessments was $51.19 \mathrm{mg} / \mathrm{dl}$. Statistical operation suggests significant decrease in total cholesterol level with the treatment $(\mathrm{P}<.001)$. Similar decreasing trend is seen in serum triglyceride $(\mathrm{P}<.001)$ and LDL cholesterol $(\mathrm{P}<.001)$. However HDL cholesterol remained similar across two measurements. $(p>.05)$

\section{Safety of fluvastatin}

Safety profile of fluvastatin treatment was assessed by comparing aminotransferase before and after fluvastatin treatment through paired t test, with a view to assess the risk for reversible elevation of liver transaminases. Neither Alanine aminotransferase (ALT) nor the Aspartate aminotransferase (AST) showed statistically significant rise after 3 months treatment of fluvastatin ( $p>05)$.

Safety of the treatment was assessed based primarily the opinion of the physician concerned. A total 7(4.3\%) of the total 162 participant had their treatment interrupted. Among the seven patients with interrupted treatment 1 (14.3\%) had to cease treatment due to lack of efficacy, 1 (14.3\%) experienced adverse event, 2 didn't return to follow-up and 3 (42.9\%) patients requested their physician to cease.

\section{Discussion:}

Among the available statins, Fluvastatin is the only synthetic molecule, a second generation, potent competitive inhibitor of 3-hydroxy-3-methylglutaryl coenzyme-A reductase, the rate-limiting enzyme in cholesterol biosynthesis. and has emerged as an effective therapeutic option for treatment of elevated cholesterol levels ${ }^{3}$ which in turn contribute to the reduction of major coronary events and death in a wide range of individuals at risk for these events. 
Mounting evidence from randomized clinical trials with statins has shown significant reduction in the incidence of CHD. ${ }^{18,19,20}$ These Trials were conducted with different available statins and demonstrated statistically significant beneficial effect on reducing cholesterol level as well the reduction of incidence of mortality and morbidity from wide range of cardiovascular causes. Systematic review of RCT by LaRosa ${ }^{21}$ also substantiated the efficacy of statins as reducer of cholesterol level eventually as a cardio protective agent. In their study pooled analysis of around 30,000 patients' data revealed evidence of $28 \%$ reduction in LDL-cholesterol, 31\% reduction of coronary events and $29 \%$ of death due to coronary heart disease. Apart from coronary heart disease a significant reduction in stroke was also reported in major clinical trials with statins in the review.

When used in conjunction with a fat-and cholesterol-restricted diet, it shows marked efficacy in treatment of hypercholesterolemia and mixed hypercholesterolemia. ${ }^{22}$ Several clinical outcome trials have also shown that substantial benefits are associated with treatment with fluvastatin in diverse populations.

Its safety profile and efficacy was not assessed in Bangladeshi population, a population significantly different from Caucasian, Negrito and other racial variations. Authors intension was to evaluate the safety and efficacy of fluvastatin in the specified population and in its registered indication in treating patients with hyperlipidemia and or mixed dyslipidemia with risk factors of cardiovascular disease in Bangladesh population.

Participants were treated with Fluvastatin $80 \mathrm{mg}$ once daily. Among the different formulations available in the market Fluvastatin XL 80 mg once daily was chosen and interventional regimen. Different formulations of fluvastatin available in the market have shown nonlinear pharmacokinetics at doses of greater than 20 mg. ${ }^{23,24}$ Consequently, there are greater than expected systemic drug concentrations in patients receiving higher doses of fluvastatin, which may lead to increased risk of adverse events, and in particular, of dose-related musculoskeletal and hepatic toxicities that are associated with this class of drugs. The greater-than-expected increase in systemic bioavailability at high doses may be due to saturable first-pass hepatic metabolism of fluvastatin. ${ }^{25}$ Increasing the duration of hepatic exposure to fluvastatin through slower delivery may decrease the systemic drug levels, which may improve tolerability at higher doses while maintaining the drug's dose-ordered efficacy. Hence, extended-release matrix tablet formulation of fluvastatin $80 \mathrm{mg}$ has been chosen to ensure sustained release of fluvastatin over 8-12 h, resulting in lower systemic drug levels as proposed by Ballantyne, McKenney and Trippe. ${ }^{26}$

All the patients were assessed twice, once at the baseline and once upon completion of three month course of treatment. Of the 162 patients enrolled in the study, around seventy percent were male and more than seventy percent were aged between 40-59 years. With a view to estimate the cardiovascular risk reduction adult subjects with registered indication of fluvastatin, e.g. patient having hypercholesterolemia and or mixed dyslipidemia with risk factors for cardiovascular disease, namely, Hypertension, Low HDL Cholesterol, history of recent PCI and Diabetes mellitus were recruited.

Majority of the participants were overweight at baseline and at the $2^{\text {nd }}$ visit the subtle decrease in the proportion of overweight was found. At baseline four out of every five of the study participants were overweight and at the end of treatment the proportion was $77.2 \%$. Paired comparison of pre and Post treatment BMI was performed through paired $t$ test. On average only $0.41 \mathrm{~kg} / \mathrm{m}^{2}$ reduction of BMI was found with the treatment which was neither statistically significant nor clinically relevant. Evidence doesn't suggest Fluvastatin as a weight reducing agent; probably this is the rationale for employing concomitant lifestyle modification and physical exercise along with lipid lowering therapy. General physicians and cardiologists prescribe the medication in combination with diet and exercise.

Blood pressure was measured at both the assessment, A systolic BP $>140 \mathrm{mmHg}$ or diastolic $\mathrm{BP}>90 \mathrm{mmHg}$ was considered as hypertension. At the commencement of the study the proportion of hypertension measured was $38.3 \%$ and at the $2^{\text {nd }}$ visit the proportion dropped sharply to $6.2 \%$ and the decrease was found to be highly significant $(\mathrm{P}<.01)$. High prevalence of hypertension among 
hypercholesterolemic patients is not surprising, it would rather support a plausible explanation coexistence of several metabolic disease like diabetes, Obesity, hypertension etc. in patient of metabolic syndrome.

Even sharp reduction in the proportion of hypertension doesn't necessarily denote the antihypertensive effect of Fluvastatin, reason for such decrease would be the concomitant use of antihypertensive. Patient diagnosed as hypertensive by the physician at baseline, were rationally prescribed necessary antihypertensive along with the lipid lowering agent. Although, to avoid contamination of results, Patients having concomitant therapy with other antihyperlipidemics or drugs with the potential of confounding effect in judgment of the treating physician were dropped. However the use of antihypertensive was not prohibited.

Efficacy of fluvastatin treatment was assessed by assessing the lipid lowering capability of the drug by comparing after treatment lipid profile with the baseline. Serum total cholesterol was found to be significantly reduced and across two assessments the reduction was 51.2 units. Average reduction in LDL-cholesterol was around 40 units. Most significant reduction was seen in serum LDL

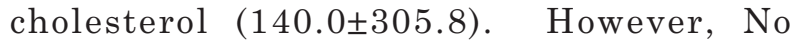
statistically significant reduction was seen in HLD cholesterol.

$\mathrm{n}$ study by Langtry et $\mathrm{al}^{27}$ fluvastatin has been shown to decrease serum LDL-C, total cholesterol and triglyceride levels, and to elevate HDL-C. However in our study the level of HDL-cholesterol remained static. Another similar study like ours ${ }^{28}$ evaluated the effects of fluvastatin XL $80 \mathrm{mg}$ on the lipid profile and showed statistically significant improvement with fluvastatin treatment in mean total cholesterol, low-density lipoprotein (LDL) cholesterol, high-density lipoprotein (HDL) cholesterol, and triglyceride values over the duration 3 and 6 months. Although our study only could reveal treatment efficacy of 3 month duration, the Di Lullo ${ }^{28}$ study confirms the lipid lowering effect of relatively longer duration, which justifies effect of longer duration of treatment. A prospective study of wider time span could elucidate further evidence.
Although fluvastatin exerts effect on all three bad cholesterol, most study highlighted its effect on LDL-cholesterol. Zavoral et al. ${ }^{29}$ demonstrated in a long-term, open-label study that fluvastatin lowered LDL-C by $25 \%$ to $31 \%$. In a pooled analysis ${ }^{30}$ of data of three RCT showed that LDLC-lowering effect of fluvastatin was apparent after 2 weeks of treatment, and the full effect was observed within 4 weeks of treatment initiation. After 4 weeks of treatment with fluvastatin XL 80 mg HS, LDL-C levels were reduced by a mean of $36.3 \%$.

Fluvastatin inhibits the progression of lesions in the coronary arteries, as evidenced by the Lipoprotein and Coronary Atherosclerosis Study (LCAS), ${ }^{31}$ the Fluvastatin Angiographic Restenosis

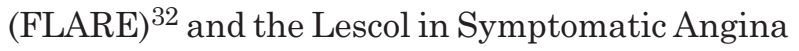
$(\text { LISA })^{33}$ trials. Therefore, it has been shown that fluvastatin is beneficial in the secondary prevention of coronary atherosclerosis. More recently, März et al. ${ }^{34}$ showed that fluvastatin causes a shift in LDL-C subfractions toward more buoyant, less atherogenic LDL particles in post-menopausal women with combined hyperlipidemia both in primary and in secondary prevention. This may explain the greater antiatherogenic effect of fluvastatin than expected based on changes in serum lipids alone.

Tolerability was assessed by evaluating new and worsening adverse events, as well as laboratory abnormalities, including alanine aminotransferase (ALT) and aspartate aminotransferase (AST). Comparison of aminotransferase level was done before and after fluvastatin treatment through paired t test, with a view to assess the risk for reversible elevation of liver transaminases. Neither Alanine aminotransferase (ALT) nor the Aspartate aminotransferase (AST) showed statistically significant rise after 3 months treatment of fluvastatin. $(\mathrm{P}>.05)$. The result suggests no significant liver enzyme increase in the study participants, although elevation of liver aminotransferase has been considered as an adverse effect of statin therapy and this has been recorded as a contraindication of such drugs. However study by Ballantyn ${ }^{30}$ also didn't notice an increase in ALT or AST.

Evaluation of adverse event of the treatment was assessed based on the opinion of the physician 
concerned. A potential limitation of our study was that for safety data we had to depend on the turnout of patient at final follow-up. However in the current study only $7(4.1 \%)$ patient were excluded due to incompleteness of data or loss to follow-up before deciding final sample. The figure was not bigger enough to impede the generalizability of study finding. At final follow-up, in addition to repetition of the baseline parameters and laboratory investigations, data on Safety of the drug was collected by inquiring and recording all adverse events or serious adverse events. According to our data out of 162 study participant $4.3 \%$ had their treatment interrupted, of which $1(0.62 \%)$ had to cease treatment due to lack of efficacy, $1(0.62 \%)$ experienced adverse event, 2 (1.24\%) didn't return to follow-up and $3(1.86 \%)$ patients requested their physician to cease the treatment. Several studies $^{28,30}$ also showed fluvastatin treatment as effective in improving the lipid profile, and it demonstrates good safety and tolerability. A systematic review by Corsini et al ${ }^{13}$ concluded based on recent data from several clinical outcome trials that substantial benefits are associated with treatment with fluvastatin in diverse populations. They also suggested a strong benefit-to-risk profile for fluvastatin.

Our study confirms/ maintains similar stance to the fluvastatin treatment as lipid lowering modality as revelled in other population. Current study validates the use of fluvastatin XL 80mg once daily as an effective and safe lipid lowering treatment for reduction of adverse cardiac events. Current study is a quasi experimental study, not randomized, which to some extent limits generalizability of the findings, however, the intension of the researcher was to assess the safety and efficacy of the study in our population which was already recommended by large, well designed and well powered studies elsewhere in wide range of population and patient settings. Further investigation could be done preferably a phase III or IV RCT for even stronger evidences.

\section{Conclusion:}

Three month treatment with Fluvastatin XL 80 $\mathrm{mg}$ reduces most of lipid parameter of lipid profile (Total cholesterol, Triglyceride and LDL) significantly. However no significant change in HDL is demonstrated with the treatment. Although treatment with statins are said to pose risk of raising liver enzyme, current data didn't find any excess risk of increasing liver enzymes. The drug is found to be well tolerated with minimal adverse event during the course of treatment. However the drug doesn't seem to be effective in weight reduction as BMI remains almost similar in two measurements.

\section{Acknowledgement:}

The study has received an unconditional grant from, Novartis Bangladesh Limited. The interventional drug used in the study was LESCOL $\mathrm{XL}^{\circledast}$ sustained release tablet containing $80 \mathrm{mg}$ fluvastatin a research molecule from Novartis. To maintain similarity in intervention all the patients were prescribed the investigational drug of same brand.

\section{References:}

1. Murray CJ, Lopez AD. Mortality by cause for eight regions of the world: Global Bureau of Disease Study. Lancet 1997; 349: 1269-1276.

2. Martin MJ, Hulley SB, Browner WS, Kuller LH \& Wentworth D. Serum cholesterol, blood pressure and mortality: implications from a cohort of 361662 men. Lancet 1986; ii: 933-936.

3. Brown G, Albers JJ, Fisher LD, Schaefer, SM Lin JT, Kaplan C, Zhao XQ, Bisson BD, Fitzpatrick VF \& Dodge HT. Regression of coronary artery disease as a result of intensive lipid-lowering therapy in men with high levels of apolipoprotein B. N Engl J Med 1990; 323: 12891298.

4. Steinberg D, Gotto AM Jr. Preventing coronary artery disease by lowering cholesterol levels. JAMA 1999;282:2043-2050.

5. Swierzewski SJ, High cholesterol treatment.(2001) .accessed on November 2009 and available on the web http://www.cardiologychannel.com/hypercholes terolemia/treatment.shtml.

6. Simons LA. Simvastatin in severe primary hypercholesterolemia: efficacy, safety, and tolerability in 595 patients over 18 weeks The principal investigators. Clin Cardiol 1993;16: 317-322.

7. Olsson AG \& Mölgaard J. The future pharmacological therapy for risk factor reduction: Hyperlipidaemia. Drugs 1988;36 (3):115-120.

8. Pignone M, Phillips C, Atkins D, Teutsch S, Mulrow C, Lohr K. "Screening and treating adults for lipid disorders". Am J Prev Med 20 (3 Suppl): 77-89.

9. U.S. Preventive Services Task Force. "Screening for Lipid Disorders: Recommendations and Rationale". http://www.ahrq.gov/clinic/ajpmsuppl/lipidrr.htm. Retrieved november 162009. 
10. Thavendiranathan P, Bagai A, Brookhart M, Choudhry N. "Primary prevention of cardiovascular diseases with statin therapy: a meta-analysis of randomized controlled trials". Arch Intern Med 2006;166 (21): 2307-13.

11. Baigent C, Keech A, Kearney PM, et al. "Efficacy and safety of cholesterol-lowering treatment: prospective meta-analysis of data from 90,056 participants in 14 randomised trials of statins". Lancet 2005;366 (9493): 1267-78.

12. Langtry HD, Markham A. Fluvastatin: a review of its use in lipid disorders. Drugs 1999; 57:583-606.

13. Corsini A, Jacobson TA and Ballantyne CM; Fluvastatin: Clinical and Safety Profile. Drugs 2004; 64 (12): 13051323.

14. Serruys PWJC, de Feyter P, Macaya C, et al., for the Lescol Intervention Prevention Study (LIPS) Investigators. Fluvastatin for prevention of cardiac events following successful first percutaneous coronary intervention: a randomized, placebo controlled trial. JAMA 2002; 287 (24): 3215-22.

15. Herd JA, Ballantyne CM, Farmer JA, et al. Effects of fluvastatin on homocysteine and serum lipids in kidney allograft recipients sclerosis Study [LCAS]). Am $J$ Cardiol 1997; 80 (3): 278-86 .

16. SPSS. SPSS for Windows 16 (Computer Software). Chicago IL: SPSS Inc; 2007.

17. StataCorp. Stata Statistical Software, version 8.2 [computer software]. College Station, TX: Stata Corporation; 2004.

18. Scandinavian Simvastatin Survival Study Group. trial of cholesterol lowering in 4444 patients with coronary heart disease: the Scandinavian Simvastatin Survival Study (4S). Lancet 1994 ; 344 (8934): 1383-9 .

19. Shepherd J, Cobbe SM, Ford I, et al., for the West of Scotland Coronary Prevention Study Group. Prevention of coronary heart disease with pravastatin in men with hypercholesterolemia. $N$ Engl J Med 1995; 333 (20): 1301-7.

20. Sacks FM, Pfeffer MA, Moye LA, et al. The effect of pravastatin on coronary events after myocardial infarction in patients with average cholesterol levels. $N$ Engl J Med 1996 Oct 3; 335: 1001-9.

21. LaRosa JC, He J, Vupputuri S. Effect of statins on risk of coronary disease: a meta-analysis of randomized controlled trials. JAMA 1999; 282 (24): 2340-46.

22. Langtry HD, Markham A. Fluvastatin: a review of its use in lipid disorders. Drugs 1999; 57:583-606.
23. Smith HT, Jokubaitis LA, Troendle AJ. Pharmacokinetics of fluvastatin and specific drug interactions. Am J Hypertens 1993;6:375S-82S.

24. Appel S, Dingemanse J. Clinical pharmacokinetics of fluvastatin with reference to other HMG-CoA reductase inhibitors. Drugs Today 1996;32(suppl A):3755 .

25. Tse FLS, Jaffe JM, Troendle A. Pharmacokinetics of fluvastatin after single and multiple doses in normal volunteers. J Clin Pharmacol 1992;32:630-8.

26. Ballantyne CM, McKenney J, Trippe BS. Efficacy and safety of an extended-release formulation of fluvastatin for once-daily treatment of primary hypercholesterolemia. Am J Cardiol 2000;86:759-63.

27. Langtry HD, Markham A. Fluvastatin: a review of its use in lipid disorders. Drugs 1999; 57 (4): 583-606 .

28. Di Lullo L, Addesse R, Comegna C, Firmi G, Galderisi C, Iannacci GR and Polito P. Effects of Fluvastatin Treatment on Lipid Profile, C-Reactive Protein Trend, and Renal Function in Dyslipidemic Patients With Chronic Renal Failure. Advances in Therapy 2005;22(6):

29. Zavoral JH, Haggerty BJ, Winick AG, et al. Efficacy of fluvastatin, a totally synthetic 3-hydroxy-3 methylglutaryl-coenzyme A reductase inhibitor. Am $J$ Cardiol 1995; 76; 37A-40A.

30. Ballantyne CM, Pazzucconi F, Pintb X, Reckless JP, Stein E, McKenney J, Bortolini M, and Chiung YT, Efficacy and Tolerability of Fluvastatin ExtendedRelease Delivery System: A Pooled Analysis. Clinical Therapeutics 2001; 23(2):

31. Herd JA, Ballantyne CM, Farmer JA, et al. Effects of fluvastatin on coronary atherosclerosis in patients with mild to moderate cholesterol elevations (Lipoprotein and Coronary Atherosclerosis Study [LCAS]). Am J Cardiol 1997; 80 (3): 278-86 .

32. Serruys PW, Foley DP, Jackson G, et al. Arandomized placebo-controlled trial of fluvastatin for prevention of restenosis after successful coronary balloon angioplasty; final results of the fluvastatin angiographic restenosis (FLARE) trial. Eur Heart J 1999; 20 (1): 58-69

33. Riegger G, Abletshauser C, Ludwig M, et al. The effect of fluvastatin on cardiac events in patients with symptomatic coronary artery disease during one year of treatment. Atherosclerosis 1999; 144 (1): 263-70 .

34. März W, Scharnagl H, Abletshauser C, et al. Fluvastatin lowers atherogenic dense low-density lipoproteins in postmenopausal women with the atherogenic lipoprotein phenotype. Circulation 2001; 103 (15): 1942-8 . 